\title{
Establishment of a system for monitoring endoplasmic reticulum redox state in mammalian cells
}

\author{
Kohsuke Kanekura ${ }^{1,7}$, Shinsuke Ishigaki ${ }^{2,7}$, Philip I Merksamer ${ }^{3}$, Feroz R Papa ${ }^{3,4,5,6}$ and Fumihiko Urano ${ }^{1}$
}

The endoplasmic reticulum (ER) performs a critical role in the oxidative folding of nascent proteins, such that perturbations to ER homeostasis may lead to protein misfolding and subsequent pathological processes. Among the mechanisms for maintaining ER homeostasis is a redox regulation, which is a critical determinant of the fate of ER-stressed cells. Here, we report the establishment of a system for monitoring the ER redox state in mammalian cells. The new ER redox-sensing system was developed based on the previously described monitoring system in yeast. Our system could successfully monitor the dynamic ER redox state in mammalian cells. Using this system, we find that manipulation of ER oxidases changes the ER redox state. The mammalian ER redox-sensing system could be used to study the mechanisms of ER redox regulation and provide a foundation for an approach to develop novel therapeutic modalities for human diseases related to dysregulated ER homeostasis including diabetes, neurodegeneration, and Wolfram syndrome.

Laboratory Investigation (2013) 93, 1254-1258; doi:10.1038/labinvest.2013.112; published online 16 September 2013

KEYWORDS: beta cell; diabetes; ER stress; neurodegeneration; neuron; redox regulation; Wolfram syndrome

The endoplasmic reticulum (ER) participates in many important cellular tasks, including protein and lipid biosyntheses, calcium regulation, redox regulation, cell signaling, and cell death. Given the many vital and complex functions of the ER, it is of little wonder that its failure can trigger a range of diseases including diabetes, neurodegeneration, and Wolfram syndrome- a genetic form of diabetes and neurodegeneration. ${ }^{1-6}$ The major mechanisms for the maintenance of ER homeostasis include redox state regulation and activation of the unfolded protein response (UPR), an adaptive response to the accumulation of misfolded proteins in the ER. ${ }^{1,7}$

The redox potential of the ER is continually preserved as an oxidizing environment to facilitate the oxidative process of disulfide-bond formation during protein folding. ${ }^{7}$ In contrast, the UPR is an on-demand, adaptive regulatory response that controls diverse processes including protein folding, protein degradation, and ER biogenesis. ${ }^{1}$ In yeast cells, it has been shown that ER redox regulation and the UPR are tightly connected, and disruption of the ER redox state triggers the
UPR and vice versa. ${ }^{8}$ However, in mammalian cells, the coordination of ER redox regulation and the UPR and their specific contributions to cell survival or death are yet to be elucidated because of the lack of a system for real-time monitoring of the ER redox state. To address this critical gap in our understanding of the mechanisms for maintenance of ER homeostasis, we developed a system for real-time monitoring of the ER redox state in mammalian cells.

\section{MATERIALS AND METHODS Reagents}

Glucose, dithiothreitol (DTT), 4-acetamido-4'-maleimidylstilbene-2,2'-disulfonic acid (AMS), and cycloheximide were obtained from Sigma-Aldrich (St Louis, MO, USA). RPMI1640 and DMEM were obtained from Invitrogen (Carlsbad, CA, USA). Short-hairpin RNA (shRNA) constructs (TRC) were obtained from RNAi core of University of Massachusetts Medical School. Anti-green-fluorescent protein (GFP) antibody was purchased from Santa Cruz Biotechnology (Santa Cruz, CA, USA).

'Division of Endocrinology, Metabolism, and Lipid Research, Department of Medicine and Department of Pathology and Immunology, Washington University School of Medicine, St Louis, MO, USA; ${ }^{2}$ Department of Neurology, Nagoya University Graduate School of Medicine, Nagoya, Japan; ${ }^{3}$ Department of Medicine, University of California San Francisco, San Francisco, CA, USA; ${ }^{4}$ Diabetes Center, University of California San Francisco, San Francisco, CA, USA; ${ }^{5}$ Lung Biology Center, University of California San Francisco, San Francisco, CA, USA and ${ }^{6}$ California Institute for Quantitative Biosciences, University of California San Francisco, San Francisco, CA, USA Correspondence: Dr F Urano, MD, PhD, Division of Endocrinology, Metabolism, and Lipid Research, Department of Medicine, Washington University School of Medicine, Saint Louis, MO 63110, USA.

E-mail: urano@dom.wustl.edu

${ }^{7}$ These authors contributed equally to this work.

Received 15 July 2013; revised 31 July 2013; accepted 3 August 2013 
a

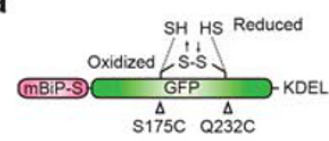

b

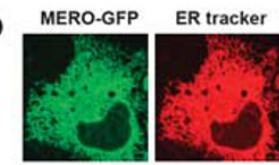

f
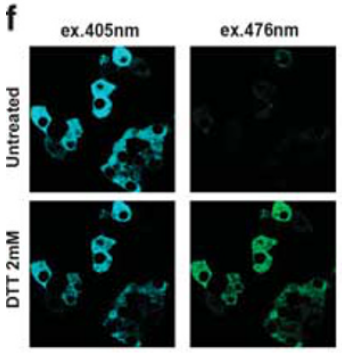

C Excitation spectrum (Em. $510 \mathrm{~nm})$

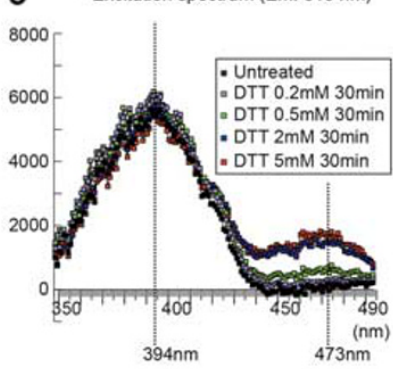

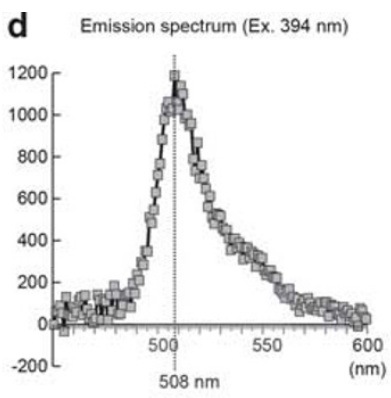
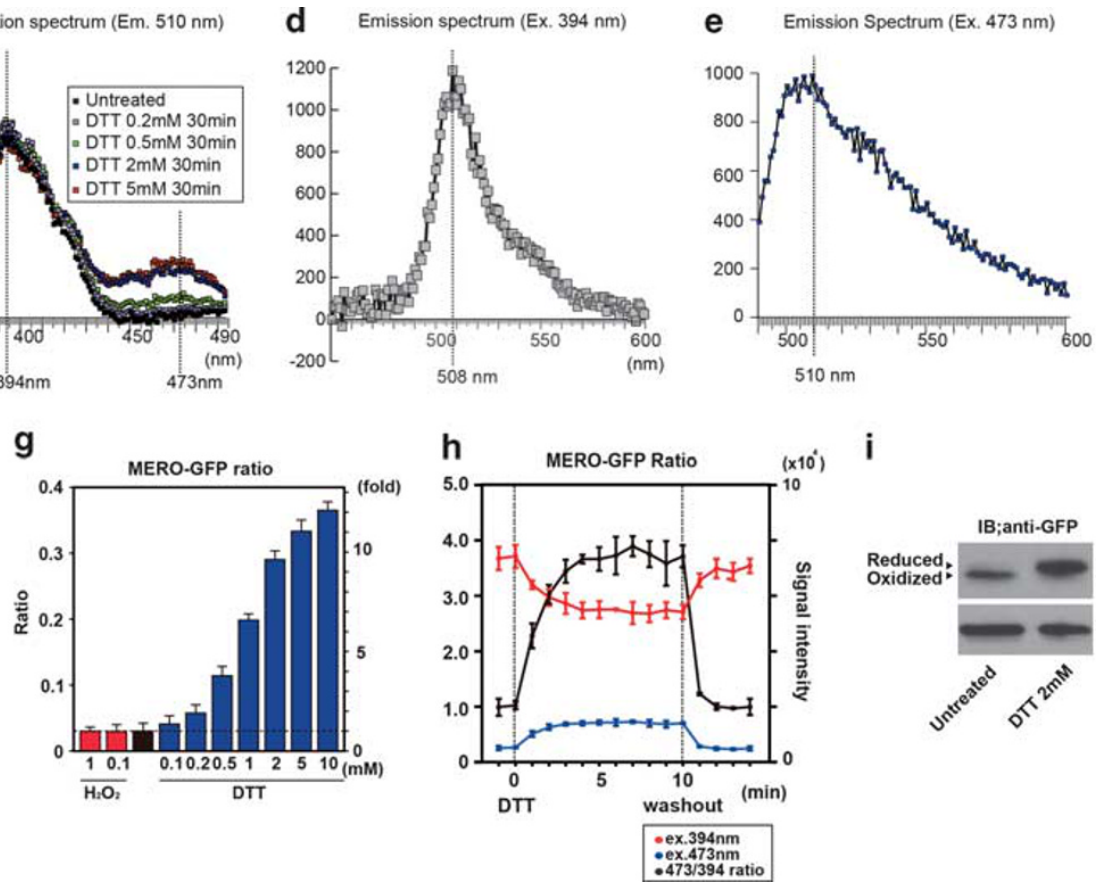

i

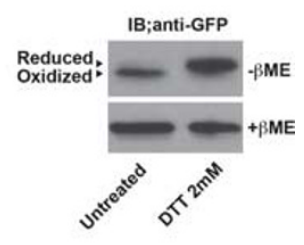

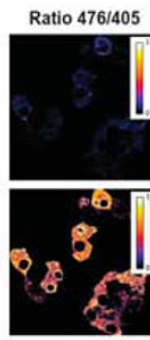

Figure 1 Characterization of MERO-GFP (Mammalian endoplasmic reticulum-localized redox-sensitive green-fluorescent protein). (a) Schematic structure of MERO-GFP. (b) Confocal imaging of MERO-GFP in COS7 cells. ER was visualized using DsRed-ER tracker. (c) Excitation spectra of MERO-GFP treated with different concentrations of dithiothreitol (DTT). (d) Emission spectrum of oxidized MERO-GFP in untreated NSC34 cells. The wavelength of excitation was $394 \mathrm{~nm}$. (e) Emission spectrum of reduced MERO-GFP in NSC34 cells treated with $2 \mathrm{mM}$ DTT. The wavelength for excitation was $473 \mathrm{~nm}$. (f) Real-time imaging of MERO-GFP signal from $476 \mathrm{~nm}$ excitation and $405 \mathrm{~nm}$ excitation in INS-1 832/13 cells treated with or without $2 \mathrm{mM}$ DTT. 476 / 405 ratio images are displayed in false colors in the lookup table. (g) MERO-GFP ratio in cells treated with the indicated concentrations of DTT and $\mathrm{H}_{2} \mathrm{O}_{2}$. MERO-GFP ratio was determined by a plate reader. Data represent means \pm s.d. from three independent experiments. (h) Real-time monitoring of MERO-GFP ratio in live NSC34 cells treated with DTT. NSC34 cells expressing MERO-GFP were treated with DTT (1 mM), and the fluorescence from MERO-GFP was measured every minute. Ten minutes after the treatment, DTT was washed out. The red line indicates the fluorescence intensity with excitation at $394 \mathrm{~nm}$. The blue line indicates the fluorescence intensity with excitation at $473 \mathrm{~nm}$. Black line indicates the normalized MERO-GFP ratio. (i) AMS (4-acetamido-4'-maleimidylstilbene-2,2'-disulfonic acid)-modified SDS-PAGE of MERO-GFP. All error bars shown, \pm s.d.

\section{Plasmid Constructions}

For constructing MERO-GFP (Mammalian ER-localized Redox-sensitive GFP) plasmid, we appended the signal sequence of mouse $\mathrm{BiP}$ and the mammalian ER-retrieval signal, KDEL, to the $\mathrm{N}$ - and $\mathrm{C}$-termini of the redox-sensitive green-fluorescent protein (GFP)-1, respectively.

\section{Cell Culture}

INS-1 832/13 cells were cultured in the RPMI-1640 containing $10 \%$ fetal bovine serum (FBS), penicillin and streptomycin, sodium pyruvate, and $0.1 \% \beta$-mercaptoethanol. Human embryonic kidney 293a (HEK293a) and NSC34 cells were cultured in DMEM containing 10\% FBS and penicillin and streptomycin. For establishing stable cell lines, cells were transduced with lentivirus expressing MERO-GFP and selected with puromycin or neomycin.

\section{FACS Analyses}

For flow cytometry analyses, INS-1 832/13 and HEK293A expressing MERO-GFP were plated onto 12-well plates, treated with each compound for indicated times, and then harvested by trypsinization. After washing with PBS, cells were resuspended in the PBS. Flow cytometry analyses were performed with LSRII (BD) at the FACS core facility of the University of Massachusetts Medical School and Washington University School of Medicine. The results were analyzed using FlowJo ver.7.6.3.

\section{Short Hairpin RNA}

Control shRNA vector: pLKO.1-puro (Addgene, Cambridge, MA, USA). shERO1L: TRCN0000059755, 5'-GCCAGAAAG TGGACCTAGTTA-3' (Broad Institute, Cambridge, MA, USA). shPRDX4: TRCN0000064821, 5'-GCTGTGATCGATG GAGAATTT-3' (Broad Institute).

\section{Quantitative Real-Time PCR}

Total RNA was extracted using RNeasy kits (Qiagen). Reverse transcriptase PCR was performed using ImPromII (Promega) reverse transcriptase, and quantitative PCR was performed with Bio-Rad iQ5 using SYBR green dye.

\section{Plate Reader}

The excitation spectra and emission spectra of MERO-GFP were determined using SAFIRE (Tecan). INS-1 832/13 cells 

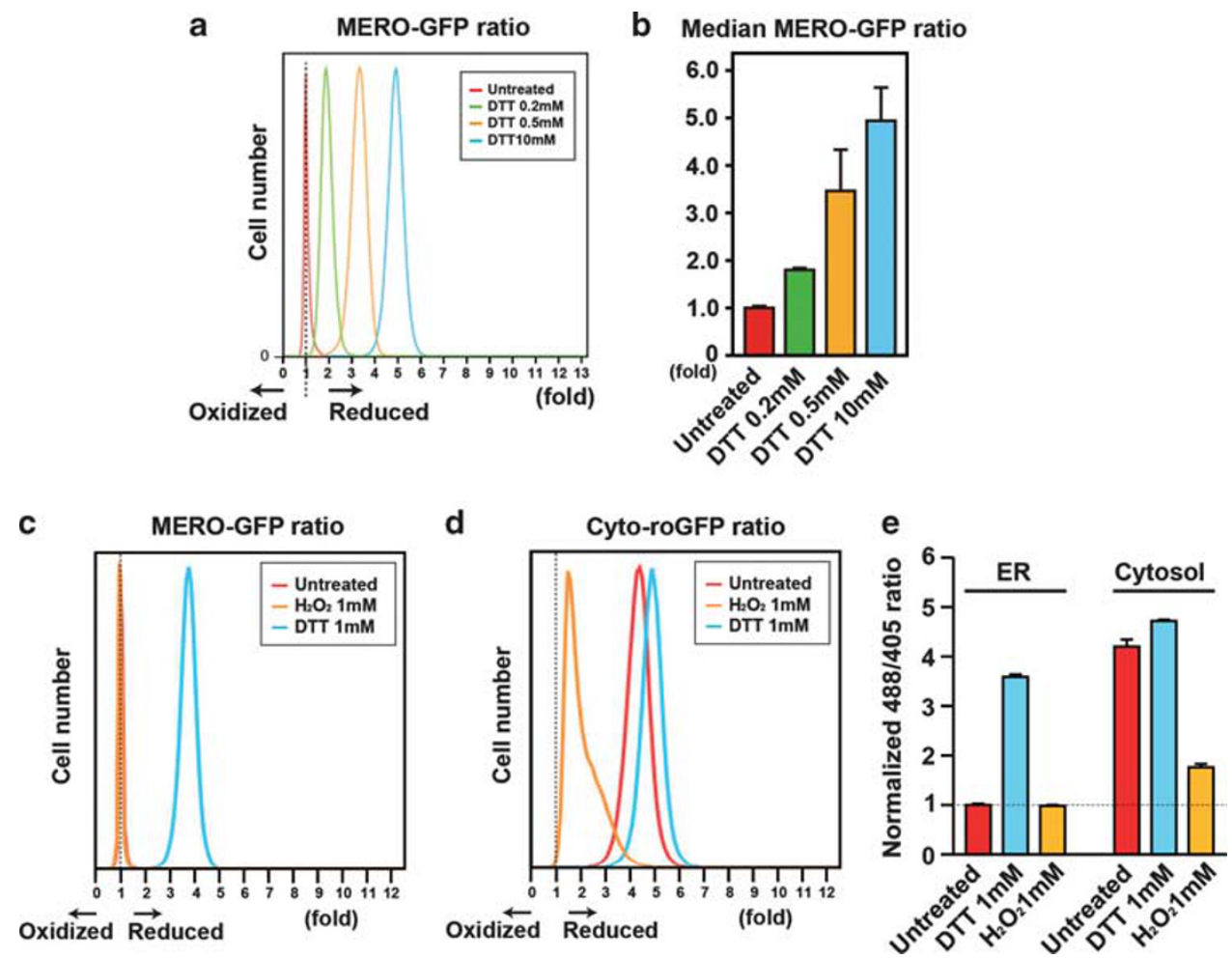

Figure 2 MERO-GFP (Mammalian endoplasmic reticulum-localized redox-sensitive green-fluorescent protein) is fully oxidized under normal conditions and is reduced by dithiothreitol (DTT) in vivo. (a) Histogram of INS-1 832/13 cells stably expressing MERO-GFP treated with DTT. The $x$ axis indicates the MERO-GFP ratio and the $y$ axis indicates the number of cells. All error bars shown, \pm s.d. (b) The median MERO-GFP ratio of the data shown in panel $\mathbf{a}$. (c) Histogram of MERO-GFP ratio of untreated cells or cells treated with $1 \mathrm{mM} \mathrm{H}_{2} \mathrm{O}_{2}$ or $1 \mathrm{mM} \mathrm{DTT}$. (d) Histogram of cytosolic roGFP (cyto-roGFP) ratio of untreated cells or cells treated with $1 \mathrm{mM} \mathrm{H}_{2} \mathrm{O}_{2}$ or $1 \mathrm{mM} \mathrm{DTT}$. (e) The median of MERO-GFP or cytosolic roGFP ratio of untreated cells or cells treated with $1 \mathrm{mM} \mathrm{H} \mathrm{O}_{2}$ or $1 \mathrm{mM} \mathrm{DTT}$.

were plated onto a 96-well plate at 50000 cells per well, and the emission spectra and excitation spectra were scanned using SAFIRE in 1-nm increments. Working range of MEROGFP was also determined using SAFIRE. INS-1 832/13 cells were plated onto 96-well plates, treated with $\mathrm{H}_{2} \mathrm{O}_{2}$ or DTT at various concentrations for $30 \mathrm{~min}$, and then the fluorescence at excitation wavelength $473 \mathrm{~nm}$ and emission wavelength $510 \mathrm{~nm}$ (for reduced MERO-GFP) or at excitation wavelength $394 \mathrm{~nm}$ and emission wavelength $510 \mathrm{~nm}$ (for oxidized MERO-GFP) was measured. After subtraction of background signal, the MERO-GFP ratio was determined.

\section{AMS-Modified SDS-PAGE}

INS-1 832/13 cells untreated or treated with 2 mM DTT were lysed with $1 \times$ SDS-PAGE sample buffer containing $25 \mathrm{mM}$ AMS with or without $\beta$-mercaptoethanol, boiled at $95^{\circ} \mathrm{C}$ for $10 \mathrm{~min}$, and applied to SDS-PAGE followed by immunoblotting with anti-GFP antibody.

\section{RESULTS}

To visualize the ER redox state in mammalian cells, we adapted the previously described monitoring system in yeast for use in mammalian cells. ${ }^{8}$ To establish an ER-specific redox sensor, we appended the signal sequence of mouse BiP and the mammalian ER-retrieval signal, KDEL, to the $\mathrm{N}$ - and C-termini of the redox-sensitive GFP, respectively (Figure 1a). $.8,10$ This recombinant protein, termed MEROGFP (Mammalian ER-localized Redox-sensitive GFP), is localized to the ER following transient transfection (Figure 1b). MERO-GFP displayed distinct excitation spectra in the fully oxidized and reduced species, with maxima at 394 and $473 \mathrm{~nm}$, respectively (Figure 1c). The emission spectra from two distinct excitation wavelengths, $508 \mathrm{~nm}$ (Figure 1d) and $510 \mathrm{~nm}$ (Figure 1e), were comparable. Confocal microscopic analysis confirmed that fluorescence from the 476-nm excitation peak significantly increased, whereas fluorescence from 405-nm excitation slightly decreased in cells treated with the strong reducing agent DTT. As a result, the ratio of the fluorescence from excitation 476 versus $405 \mathrm{~nm}$ significantly increased (Figure 1f). The fluorescence ratio between excitation 488 versus $405 \mathrm{~nm}$ normalized to wild-type, untreated cells is called the MERO-GFP ratio. Treatment with $\mathrm{H}_{2} \mathrm{O}_{2}$, a strong oxidizing agent, did not change the MERO-GFP ratio, indicating that MERO-GFP is almost $100 \%$ oxidized in vivo, consistent with the highly oxidizing environment of the ER (Figure $1 \mathrm{~g}$ ). In contrast, DTT treatment increased the MERO-GFP ratio in a dosedependent manner, as expected (Figure 1g). Next, we 
a

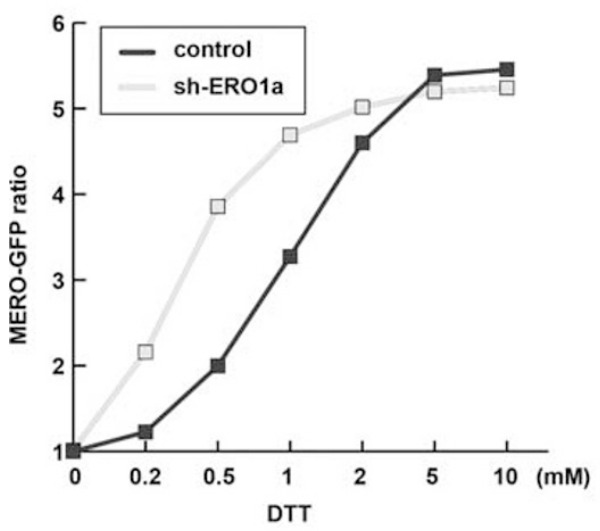

b

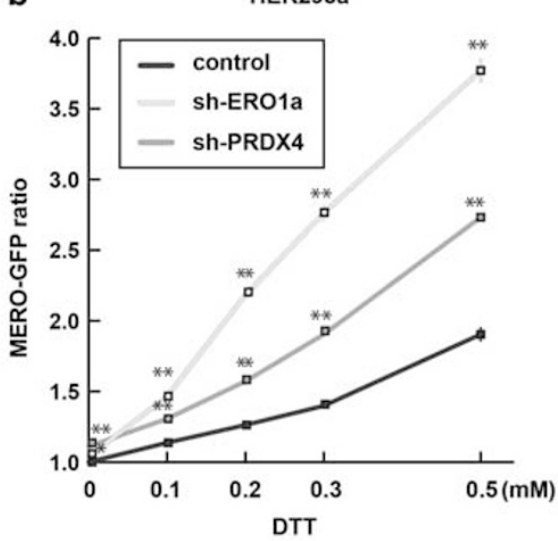

c

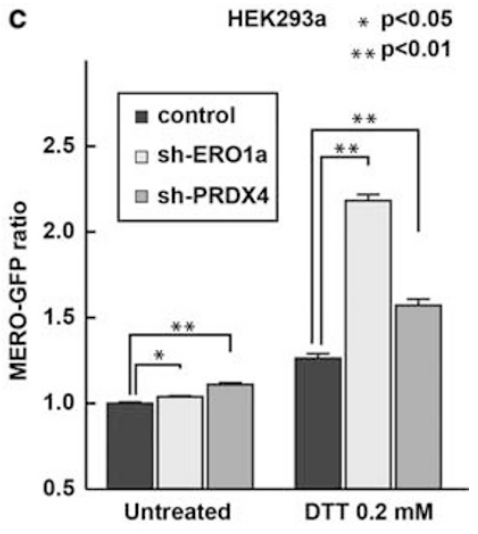

d
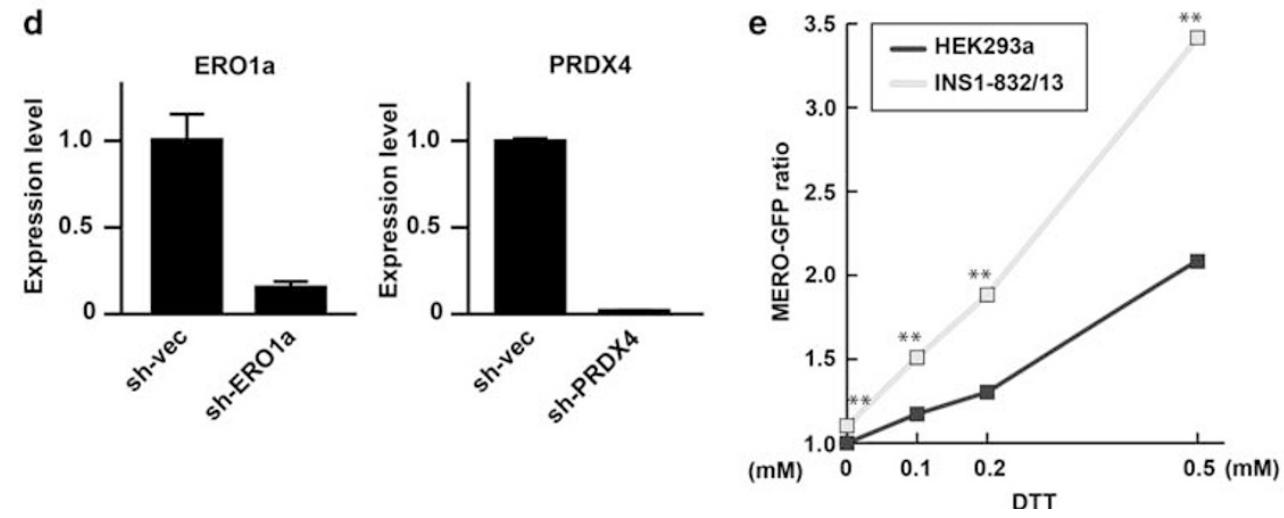

Figure 3 MERO-GFP (Mammalian endoplasmic reticulum-localized redox-sensitive green-fluorescent protein) monitors the dynamic ER redox state in mammalian cells. (a) The MERO-GFP ratio in HEK293a cells transduced with control shRNA or shRNA directed against ERO1 $\alpha$ and then treated with different concentrations of dithiothreitol (DTT). (b) The MERO-GFP ratio in HEK293a cells transduced with control shRNA, shRNA directed against ERO1 $\alpha$, or shRNA directed against PRDX4, and then treated with different concentrations of DTT. (c) The MERO-GFP ratio of HEK293a cells transduced with control shRNA, shRNA directed against ERO1 $\alpha$, or shRNA directed against PRDX4, and treated with or without $0.2 \mathrm{mM}$ DTT. (d) Expression levels of ERO1 $\alpha$ and PRDX4 mRNA in HEK293a cells transduced with control shRNA or shRNA directed against ERO1 $\alpha$ (left panel) or PRDX4 (right panel). Expression levels of mRNA were measured using real-time PCR. (e) The oxidizing capacity of the ER in different cell lines. All error bars shown, \pm s.d. ${ }^{*} P<0.05,{ }^{* *} P<0.01$.

monitored the MERO-GFP ratio in DTT-treated cells at different time points. Within a few minutes of treatment with DTT, the ER was fully reduced and returned to an oxidized environment within a minute of DTT washout (Figure 1h). To confirm that the MERO-GFP ratio reflected changes in its redox state in vivo, we monitored the redox state of MEROGFP using non-reducing SDS-PAGE in combination with the thiol-alkylating reagent, AMS. As expected, non-reducing SDS-PAGE of lysates from untreated cells showed a rapidly migrating band of MERO-GFP, whereas lysates from DTTtreated cells showed only one slowly migrating form of MERO-GFP. These results indicate that MERO-GFP is fully oxidized in the ER in vivo and becomes fully reduced following DTT treatment (Figure 1i). These results propose that MERO-GFP can be used for real-time, live-monitoring of the redox state within the ER of mammalian cells.

To precisely monitor the MERO-GFP ratio in vivo, we used flow cytometry to measure the ratio of fluorescence from excitation at 488 versus $405 \mathrm{~nm}$. DTT treatment increased the MERO-GFP ratio in the pancreatic $\beta$ cell line, INS-1 832/13 cells, in a dose-dependent manner (Figures 2a and b). Although DTT treatment increased the MERO-GFP ratio, $\mathrm{H}_{2} \mathrm{O}_{2}$ treatment did not change the MERO-GFP ratio (Figures $2 \mathrm{c}$ and $\mathrm{e}$ ), indicating that MERO-GFP was almost fully oxidized in the ER at basal levels. Compared with MERO-GFP, cytosolic roGFP (cyto-roGFP) was almost fully reduced at the basal level and was reduced and oxidized by treatments with DTT and $\mathrm{H}_{2} \mathrm{O}_{2}$, respectively (Figures $2 \mathrm{~d}$ and e).

To assess whether MERO-GFP monitors the dynamic ER redox state in mammalian cells, we manipulated the expression of the key regulators of ER redox state and then monitored the ER redox state in mammalian cells stably expressing MERO-GFP. ER oxidoreductin- $1 \alpha($ ERO1 $\alpha)$ is an ER-resident oxidase and one of the major regulators of the ER redox state. ${ }^{7,11}$ Suppression of ERO1 $\alpha$ expression by shRNA directed against ERO1 $\alpha$ increased the MERO-GFP ratio in HEK293a cells treated with DTT in a dose-dependent manner (Figures 3a-d). Suppression of another ER-resident oxidase, Peroxiredoxin IV (PRDX4), ${ }^{12}$ by shRNA also increased the MERO-GFP ratio in HEK293a cells treated 
with DTT in a dose-dependent manner (Figures 3b-d). These results indicate that the suppression of ER-resident oxidases impairs oxidizing capacity of the ER to maintain ER redox homeostasis. The difference between control cells and ERO1 $\alpha$ - or PRDX4-knockdown cells was enhanced in the presence of DTT, suggesting that the oxidizing capacity of the ER can be precisely assessed when cells are challenged with a reducing agent such as DTT.

To further examine the ER redox regulation in different types of mammalian cells, we monitored the MERO-GFP ratio in HEK293a cells and INS-1 832/13 cells, a rodent $\beta$ cell line, treated with different concentrations of DTT. The results shown in Figure 3e indicate that INS-1 832/13 cells are more susceptible to DTT-mediated reduction of the ER as compared with HEK293a cells. Collectively, these results indicate that MERO-GFP can monitor the dynamic ER redox state in different types of mammalian cells.

\section{DISCUSSION}

Growing evidence indicates that dysregulation of ER homeostasis triggers a range of human chronic diseases, including diabetes, neurodegenerative diseases, and Wolfram syndrome. ${ }^{1-6,13,14}$ However, currently there is no effective therapy targeting the ER for such diseases because of the lack of a system that precisely monitors ER homeostasis. It has been established that the redox regulation is one of the major components for maintaining ER homeostasis. In this study, we report a new method for real-time monitoring of ER redox state in mammalian cells based on prior methods. Using this new method, we could confirm that ER oxidases have an important role in maintaining an oxidizing environment in the ER. We also revealed that different cell types might have different capacity of oxidative protein folding in the ER.

It has been proposed that chemical compounds that have the ability to manipulate the ER can be used for treating diseases associated with ER stress. ${ }^{15}$ We could utilize our method for identifying chemical compounds that can maintain an oxidizing environment in the ER under pathological conditions. We could also assess the roles of genes and proteins that can potentially manipulate the ER redox state. Future studies will focus on the use of this system in human samples to identify genes, proteins, and chemical compounds that can modify ER functions.

\section{ACKNOWLEDGEMENTS}

We thank Karen Sargent, Cris Brown, and Mai Kanekura for technical assistance and Agata Jurczyk, Richard Konz and Takeshi Egawa for technical advice. This work was supported by grants from NIH (DK067493, DK016746, P60 DK020579, and UL1 TR000448), JDRF (47-2012-760), ADA (1-12-CT-61), and the Jack and J.T. Snow Scientific Research Foundation to F. Urano and the Grant-in-Aid for Exploratory Research/JST to S Ishigaki. K Kanekura was partly supported by fellowships from the Japan Society for the Promotion of Science and Uehara Memorial Foundation.

\section{DISCLOSURE/CONFLICT OF INTEREST}

The authors declare no conflict of interest.

1. Walter $P$, Ron $D$. The unfolded protein response: from stress pathway to homeostatic regulation. Science 2011;334:1081-1086.

2. Scheuner D, Kaufman RJ. The unfolded protein response: a pathway that links insulin demand with beta-cell failure and diabetes. Endocr Rev 2008;29:317-333.

3. Oslowski CM, Urano F. The binary switch that controls the life and death decisions of ER stressed beta cells. Curr Opin Cell Biol 2011; 23:207-215.

4. Hotamisligil GS. Endoplasmic reticulum stress and atherosclerosis. Nat Med 2010;16:396-399.

5. Hotamisligil GS. Endoplasmic reticulum stress and the inflammatory basis of metabolic disease. Cell 2010;140:900-917.

6. Ozcan L, Tabas I. Role of endoplasmic reticulum stress in metabolic disease and other disorders. An Rev Med 2012;63:317-328.

7. Tu BP, Weissman JS. Oxidative protein folding in eukaryotes: mechanisms and consequences. J Cell Biol 2004;164:341-346.

8. Merksamer PI, Trusina A, Papa FR. Real-time redox measurements during endoplasmic reticulum stress reveal interlinked protein folding functions. Cell 2008;135:933-947.

9. Dooley CT, Dore TM, Hanson GT, et al. Imaging dynamic redox changes in mammalian cells with green fluorescent protein indicators. J Biol Chem 2004;279:22284-22293.

10. Hanson GT, Aggeler R, Oglesbee D, et al. Investigating mitochondrial redox potential with redox-sensitive green fluorescent protein indicators. J Biol Chem 2004;279:13044-13053.

11. Cabibbo A, Pagani M, Fabbri M, et al. ERO1-L, a human protein that favors disulfide bond formation in the endoplasmic reticulum. J Biol Chem 2000;275:4827-4833.

12. Zito $E$, Melo EP, Yang $Y$, et al. Oxidative protein folding by an endoplasmic reticulum-localized peroxiredoxin. Molecular cell 2010; 40:787-797.

13. Fonseca SG, Fukuma M, Lipson KL, et al. WFS1 is a novel component of the unfolded protein response and maintains homeostasis of the endoplasmic reticulum in pancreatic \{beta\}-cells. J Biol Chem 2005;280: 39609-39615.

14. Fonseca SG, Ishigaki S, Oslowski CM, et al. Wolfram syndrome 1 gene negatively regulates $E R$ stress signaling in rodent and human cells. T J Clin Investig 2010;120:744-755.

15. Ozcan U, Yilmaz E, Ozcan L, et al. Chemical chaperones reduce ER stress and restore glucose homeostasis in a mouse model of type 2 diabetes. Science 2006;313:1137-1140. 